\title{
Incidencia del bienestar subjetivo y equilibrio trabajo-familia en cuanto a la cultura trabajo-familia en trabajadores del poder judicial chileno
}

\section{Incidence of subjective well-being and work-family balance in terms of work- family culture in workers of the chilean judicial branch}

Andrés Jiménez-Figueroa

Universidad de Talca,Talca, Chile
Verónica Gómez-Urrutia

Universidad Autónoma de Chile, Talca, Chile

\section{Resumen}

El presente trabajo determina la relación entre bienestar subjetivo y equilibrio trabajo-familia en la cultura trabajo-familia en trabajadores ligados al poder judicial en Chile. A 143 trabajadores se les administró el Cuestionario de Cultura Trabajo-Famila (Thompson et al., 1999), la Escala de Equilibrio Trabajo-Famila (Moreno et al., 2009) y Bienestar Subjetivo (Diener et al., 1999). Se observa que la cultura trabajo-familia es explicada en un $44.7 \%$ por el bienestar subjetivo y específicamente con la Interfase Negativa del Equilibrio Trabajo-Familia. Se concluye con las implicancias en la gestión organizacional para aplicar estrategias organizacionales que incorporen las dimensiones de bienestar y políticas de conciliación, que consideren un entorno saludable para los trabajadores del poder judicial.

Palabras clave: cultura trabajo-familia, equilibrio trabajo-familia, bienestar, poder judicial

\section{Abstract}

This study determines the relationship between subjective well-being and work-family balance in work-family culture in workers linked to the judiciary in Chile. 143 workers were administered the Work-Family Culture Questionnaire (Thompson et al., 1999), the Work-Family Balance Scale (Moreno et al., 2009) and Subjective Well-being (Diener et al., 1984). We found that $44.7 \%$ of the Work-Family Culture is explained by subjective well-being and specifically with the Negative Interface of the Work-Family Balance. It concludes with the implications of promoting initiatives to foster organizational strategies that incorporate the dimensions of well-being and conciliation policies that build a healthy environment for workers in the judiciary.

Keywords: Work-Family Culture, Work-Family Balance, Well-Being, Judicial Branch

Andrés Jiménez-Figueroa, Universidad de Talca, Chile; Verónica Gómez-Urrutia , Universidad Autónoma de Chile, Chile.

La correspondencia en relación con este artículo se dirige a Andrés Jiménez-Figueroa, Universidad de Talca, Chile. Dirección electrónica: anjimenez@,utalca.cl 
Durante las últimas dos décadas, Chile ha sufrido una evolución de forma acelerada, que ha dado paso a cambios tanto económicos como sociales, que ha favorecido la integración de la mujer al mundo laboral. Lo anterior genera variaciones en otras áreas de la vida social de las personas como lo es la reestructuración en los "roles" en la familia, lo que ha hecho asumir transformaciones importantes en el comportamiento de sus integrantes; por su parte, a la mujer se le dificulta continuar desarrollando las mismas funciones que estaba desempeñando y además desarrollar un trabajo fuera de su hogar. Por ello, se vuelve necesaria una nueva división de las tareas y responsabilidades familiares por parte de los cónyuges, pero es de gran relevancia la conciencia y el apoyo de las empresas y organizaciones para facilitar la conciliación del trabajo y la familia (Caamaño, 2008; Faúndez et al., 2013).

En los años 1970, las temáticas de la incorporación de la mujer al mercado laboral, los hogares monoparentales y las familias con doble ingreso económico motivaron el interés por la relación entre el trabajo y la familia (Jiménez et al., 2012), puesto que la vida familiar se complica debido a que tanto hombres como mujeres están obligados en su día tras día a dedicar más tiempo a su vida laboral que a la familiar, a pesar de cumplir un horario normal de ocho horas, por lo que muchas veces el agotamiento y el estrés acumulados como producto de la jornada laboral sobrecargan en el área donde la persona se encuentra más cómoda siendo esta la familia (Gómez \& Jiménez, 2019).

Además, los diferentes roles que deben desempeñar los trabajadores, principalmente las mujeres, generan que las exigencias de las diferentes áreas se multipliquen y aumenten las exigencias emocionales y físicas (Gragnano et al., 2020). Así, se manifesta el sentirse presionados por causa de la falta de tiempo para cumplir de buena forma en sus trabajos y en su hogar, lo que conlleva a sentimientos de insatisfacción y frustración por no ejercer el control de su tiempo (Instituto Nacional de Estadística de Chile, 2017).

La vida familiar y laboral de las personas conforman y contribuyen al desarrollo integral de ellas, pues en el área familiar se desarrollan tanto social, valórica, afectiva como culturalmente; asimismo, en el ámbito laboral, adquieren productos y servicios que les permiten subsistir de forma económica, desenvolverse, socializar y, a su vez, contribuir a la sociedad con sus conocimientos, capacidades y su tiempo. Sin embargo, muchas veces, ambas áreas no se complementan y tampoco se adaptan fácilmente a las exigencias de tiempo que generan. Para que sea factible su conciliación, es necesario encontrar soluciones desde una mirada holística que involucren la política, la familia, las empresas, la sociedad y la persona como ente individual (Schmith, 2014).

En Chile este escenario se ha tornado complejo debido al contexto de jornadas laborales excesivamente largas, la desigualdad en los ingresos y las oportunidades entre hombres y mujeres, lo que afecta negativamente en el desarrollo de carrera debido a la escisión de las demandas domésticas y laborales, por lo que ambas esferas necesitan una transformación de fondo y profunda, lo que es un gran desafío para los entes actores en la conciliación trabajo y familia (Staab, 2017).

Este conflicto, que se genera a partir de la cultura trabajo y familia, involucra a diferentes actores, entre los cuales se encuentra el Estado en lo atinente a la promoción políticas laborales que impacten positivamente en la conciliación de estas áreas; además, las empresas deben ofrecer servicios que apoyen a las familias de manera eficaz al prestar atención a las nuevas necesidades de los trabajadores y a las medidas mínimas obligatorias por ley (Ministerio de Salud de Chile, 2017). 
La vida familiar constituye uno de los pilares vitales y reales accionistas en cuanto a la motivación y la productividad de los trabajadores en una organización, pues, si existe mayor satisfacción y sentimiento de equilibrio entre su vida laboral y familiar-personal, esta será una ventaja competitiva para las empresas. Sin embargo, de los beneficios que puede generar una mayor conciliación entre trabajo y familia para las organizaciones, la mayoría de estas no ofrece estrategias eficaces que permitan cubrir y conciliar estas dos áreas (Cachutt \& Ortiz, 2015).

En Chile, en las últimas décadas se ha buscado profundizar el tema y ha surgido una serie de políticas organizacionales que abordan la conciliación trabajo y familia principalmente desde la perspectiva del apoyo directivo, pues es una variable clave para su desarrollo, porque ha quedado demostrado que las políticas o las medidas adoptadas en pro de la conciliación entre el trabajo y la familia no tienen mayor efectividad si no existe en la organización una cultura y apoyo directivo persistente (Jiménez \& Gómez, 2015).

En cuanto a los funcionarios de organismos públicos de la Región del Maule, se ha evidenciado en un estudio que en relación al equilibrio trabajo y familia, percibido entre hombres y mujeres, existen diferencias significativas, puesto que las mujeres presentan un nivel mayor nivel de conflicto trabajo y familia respecto de los hombres, además de menor nivel de satisfacción en el trabajo y en el hogar; por lo tanto, se adecuan, en menor grado, a los diferentes roles con los que debe cumplir (Riquelme et al., 2012).

Además, cabe destacar que en un estudio realizado por el Servicio Nacional de la Mujer (2013) se obtuvo que el $42 \%$ de las empresas chilenas no promueve la conciliación trabajo y familia. De esta forma, el sector público corresponde a uno de los peor evaluados, puesto que solo el $38 \%$ de los encuestados manifiesta que su organización promueve la conciliación entre estas dos esferas. Entre dichas condiciones laborales, se destacan las extensas jornadas de trabajo en funcionarios del sector público, mayormente, en aquellos que pertenecen al Poder Judicial, lo cual produce como consecuencia que los trabajadores se sientan más presionados, con mayor estrés y ansiedad, lo que además genera un gran desequilibrio en el bienestar de cada uno (Brizuela, 2016).

A pesar de los problemas que conllevan las largas jornadas laborales, existe poco conocimiento sobre esta temática, además de investigaciones que exponen y ofrecen pautas por seguir ante este tipo de situaciones (Brizuela, 2016). Por lo tanto, es relevante realizar esta investigación con funcionarios del Poder Judicial, los cuales están sometidos a largas jornadas de trabajo y, de esta forma, el tiempo resulta un factor clave para el desarrollo del bienestar de los trabajadores y la disminución del conflicto en la vida familiar y laboral de estos.

Existen varias teorías que han intentado explicar la contribución de los programas de trabajo en distintas variables tales como conciliación trabajo-familia, disminución del conflicto trabajo-familia y el mejoramiento del bienestar de los empleados (McDonald et al., 2005). Sin embargo, aún no se establece el grado de la relación entre las variables, lo cual es un dato necesario para las futuras investigaciones. Se ha comparado en investigaciones que el compromiso organizacional de los empleados aumenta con las iniciativas a favor de la conciliación trabajo y familia (Smith \& Gardner, 2007).

Las variables como el apoyo percibido, la confianza en la dirección, la cultura trabajo y familia y la percepción de apoyo de las organizaciones a las familias son predictores de actitud y niveles de conflicto trabajo-familia de los empleados; por consiguiente, afecta el bienestar (Behson, 2002). Es decir, 
existe evidencia de la relación entre las variables expuestas en esta investigación; sin embargo, no existe un modelo explicativo de la relación. La cultura cumple un papel fundamental en las políticas, porque influye en cómo los empleados las perciben y el nivel de conocimiento (Servicio Nacional de la Mujer, 2003). Una forma en que el país intenta enfrentar estas transformaciones, en conjunto con el cambio en el modelo económico y el aumento del envejecimiento en la población, es mediante la acentuación de la importancia del apoyo a la conciliación trabajo-familia, por medio de las políticas públicas de corresponsabilidad familiar (Álvarez, 2015).

Un estudio realizado por Gröpel y Kuhl (2009) confirma la relación entre el equilibrio trabajo-familia y bienestar subjetivo. Los resultados del estudio señalan que el equilibrio percibido predice el nivel el bienestar subjetivo, porque si las personas perciben tener el tiempo necesario para satisfacer sus necesidades, aumenta su bienestar. De la misma forma, Greenhaus et al. (2003) en su investigación confirman la existencia de esta relación y la influencia del equilibrio trabajo-familia; es decir, el tiempo invertido en ambas áreas y el bienestar subjetivo. Además, en una investigación realizada por GrantVallone y Donaldson (2001) en la que se utilizaron autoinformes e informe de compañeros de trabajo, se evidencia que el conflicto trabajo-familia constituye un predictor longitudinal de bienestar positivo de los empleados.

Guic et al. (2006) señalan, en un estudio de centros de atención primaria, que un conflicto de roles, provocado por la falta de claridad con respecto a las labores por estamento, afecta el bienestar de los trabajadores y directamente el equilibrio entre roles; es decir, equilibrio trabajo-familia. Por lo anterior, resulta relevante la investigación en personas relacionadas con el poder judicial y cómo su contexto afecta en las variables por estudiar. Las investigaciones señalan diferencias de género en la intensidad en la que perciben variables como estrés y bienestar subjetivo en los funcionarios del poder judicial (Spielberg \& Reheiser, 1998).

Como se ha mencionado anteriormente, el equilibrio trabajo-familia se considera el punto de partida para fomentar una cultura familiar amigable en un contexto empresarial, el cual no favorece el equilibrio trabajo-familia y lo cuestiona en cuanto a la relevancia para los trabajadores (Debeljuh \& Jáuregui, 2004).

Es importante mencionar que el concepto de equilibrio se propuso como opuesto al conflicto trabajo-familia definido como conflicto interrol, en el cual las presiones del trabajo y los dominios familiares son incompatibles entre sí, porque este busca armonizar las tareas de rol familiar y laboral al establecer un equilibrio positivo (Frone, 2003). Asimismo, el equilibrio trabajo-familia cuenta con dos significados. Uno de estos centrado en la falta de conflicto entre los roles tanto del trabajo como del hogar, porque la incompatibilidad, en alguna de estas esferas, interferirá con la otra, mientras que el segundo significado está centrado en un equilibrio positivo, que facilite los roles dentro del trabajo y la familia, por lo que sí existen experiencias positivas en un dominio. El otro se interfiere por lo que se configura una clasificación de equilibrio trabajo-familia centrado en dos tipos de consecuencias, el conflicto en relación con la facilitación (Frone, 2003; Greenhaus \& Beuttel, 1985; Grzywacz \& Bass, 2003; Jiménez \& Moyano, 2008).

En presente estudio se ha formulado como hipótesis para encontrar relaciones positivas y significativas entre la cultura trabajo-familia y el equilibrio trabajo familia y entre cultura trabajo-familia y el bienestar 
subjetivo. Asimismo, se busca determinar la incidencia de las variables de bienestar subjetivo y equilibrio trabajo-familia en la variable cultura trabajo-familia y de esta forma establecer un modelo predictivo.

\section{Método}

\section{Participantes}

La muestra estuvo compuesta por un total 143 funcionarios públicos de la séptima región del Maule, con un porcentaje de $49.9 \%$ de mujeres y un $50.1 \%$ de hombres. Las edades oscilan entre los 23 y los 59 años, con un promedio de 39 años. En cuanto a su nivel de estudios, un 58.4\% es universitario, $32.8 \%$ cuenta con enseñanza a nivel técnica y el $8 \%$ solo alcanzó hasta la enseñanza secundaria. En cuanto al área familiar, el 69\% de la muestra tiene de uno a tres hijos viviendo con ellos, el $31 \%$ no tiene hijos. En este estudio, participó la totalidad de funcionarios públicos de la comuna de Talca (Chile), quienes participaron voluntariamente previo consentimiento informado. El tipo de muestra es no probabilística o dirigida, puesto que el proceso de selección se orientó de acuerdo con las características de la investigación, en lugar de un criterio más bien estadístico, al considerar como criterio que los participantes sean trabajadores del Poder Judicial de la Región del Maule.

\section{Variables e instrumentos}

Son cuatro los instrumentos administrados durante el estudio: (a) Cultura Trabajo-Familia (Thompson et al., 1999). Consta de 20 ítems en formato de respuesta likert de 7 puntos, donde el 7 representa totalmente en acuerdo y el 1 representa totalmente en desacuerdo. El cuestionario mide tres componentes de la cultura trabajo-familia: apoyo directivo, consecuencias de carrera y demandas de tiempo. La confiabilidad general del instrumento es de 0.86 , mientras que el de sus dimensiones corresponde a .85 para apoyo directivo; .79 para consecuencias de carrera (5 ítems) y .76 para demandas de tiempo, (b) Cuestionario de Interacción Trabajo-Familia, SWING (Moreno et al., 2009). Se compone por 22 ítems de escala tipo likert, con 4 alternativas de respuesta de 0 a 3 (donde 0 es nunca; 1, a veces; 2, a menudo y 3 , siempre). Los ítems están distribuidos en 4 subescalas: interacción negativa trabajo-familia (ítems del 1 al 8), interacción negativa familia-trabajo (ítems del 9-12), interacción positiva trabajo-familia (ítems del 13 al 17) e interacción positiva familia-trabajo (ítems del 18 al 22), que presenta una buena consistencia interna global de .89, (c) Escala de Satisfacción con la Vida (Diener et al.,1999). Su objetivo es la medición de la satisfacción con la vida mediante un juicio cognitivo global que hace sobre su vida quien responde. Está compuesta por un total de 5 ítems, que se contestan mediante una escala tipo Likert, donde 1 es muy en desacuerdo y 7, muy de acuerdo. Se utilizó la adaptación y la validación de Moyano y Ramos (2008), donde se obtuvo un alfa de Cronbach de .87 y un Coeficiente de Spearman Brown de .83, en estudiantes universitarios de pregrado con edades entre 19 y 31 años, siendo esta escala altamente confiable y (d) Escala de Felicidad Subjetiva (Lyubomirsky \& Lepper, 1999). Está compuesta por 4 ítems elaborados de acuerdo con una escala de diferencial semántico de 1 a 7 , donde 7 representa la mayor felicidad. Se utiliza la versión chilena con una confiabilidad alfa de Cronbach de .79 y de .77 en test-retest en una muestra de 133 participantes (Moyano \& Ramos, 2007). 


\section{Procedimiento}

Para la realización del estudio, se elaboró y se presentó un proyecto con los principales objetivos y cronograma de actividades. Luego de la correspondiente autorización, los investigadores responsables aplicaron en la institución pública de manera presencial cuatro instrumentos más una escala de caracterización sociodemográfca, los cuales fueron contestados en el periodo de julio a agosto de 2016 previa aceptación del consentimiento informado de parte de los participantes. Después, se consolidó la información de los instrumentos en una base de datos al diferenciar las dimensiones positiva y negativa de interacción trabajo-familia e insertar el valor de las respuestas.

\section{Análisis de los datos}

Se utilizaron procedimientos descriptivos, pruebas de normalidad, correlaciones bivariadas con el objetivo de describir las relaciones significativas, y modelos de regresión lineal para identificar la incidencia del bienestar subjetivo y el equilibrio trabajo-familia en la cultura trabajo-familia, mediante el programa estadístico SPSS Statistics 22.

\section{Resultados}

Según las pruebas de normalidad, se establece que las variables del estudio no poseen una distribución normal, por lo cual se procede a realizar pruebas no paramétricas (ver tabla 1).

Tabla 1

Análisis de normalidad

\begin{tabular}{lccc}
\hline \multicolumn{1}{c}{ Variables } & \multicolumn{2}{c}{ Kolmogorov-Smirnov $^{\mathrm{a}}$} \\
\hline Equilibrio Trabajo-Familia & Estadístico & gl & .200 \\
Satisfacción & .059 & 125 & .001 \\
Felicidad subjetiva & .109 & 125 & .004 \\
Cultura & .100 & 125 & .015 \\
\hline
\end{tabular}

${ }^{a}$ Corrección de la significación de Lilliefors.

En primer lugar, se sintetiza, la descripción de los niveles de cultura trabajo-familia, equilibrio trabajo-familia, bienestar subjetivo presentes en la muestra y las posibles relaciones entre las variables estudiadas a modo de responder a las hipótesis de interés.

Como se observa en la tabla 2, la variable cultura trabajo-familia posee un puntaje promedio superior a la mitad del puntaje de la escala $(\mathrm{M}=78.58, \mathrm{DE}=15.71)$. La variable equilibrio trabajo-familia posee un puntaje promedio inferior a mitad del puntaje de la escala $(\mathrm{M}=29.02, \mathrm{DE}=7.83)$. La variable satisfacción vital posee un promedio superior a la mitad del puntaje de la escala $(\mathrm{M}=24.5, \mathrm{DE}=6.43)$. La variable felicidad subjetiva posee un puntaje promedio superior a la mitad del puntaje de la escala (M $=19.14, \mathrm{DE}=3.10$ ). 
Tabla 2

Análisis estadísticos descriptivos variables cultura trabajo-familia, equilibrio trabajo-familia y bienestar subjetivo

\begin{tabular}{lccccc}
\hline \multicolumn{1}{c}{ Variables } & $\begin{array}{c}\text { Número de } \\
\text { participantes }\end{array}$ & Mínimo & Máximo & M & DE \\
\hline Cultura trabajo-familia & 125 & 32 & 134 & 78.58 & 15.716 \\
Equilibrio trabajo-familia & 125 & 10 & 51 & 29.02 & 7.831 \\
Satisfacción vital & 125 & 8 & 34 & 24.55 & 6.434 \\
Felicidad subjetiva & 125 & 11 & 27 & 19.14 & 3.105 \\
\hline
\end{tabular}

Como se observa en la tabla 3 , existe una correlación positiva baja estadísticamente significativa (positiva baja) entre Cultura Trabajo-Familia y Equilibrio Trabajo-Familia (rho $=.319$ ).

\section{Tabla 3}

Matriz de correlación entre las variables cultura trabajo-familia, bienestar subjetivo, Equilibrio Trabajo-Familia

\begin{tabular}{lccc}
\hline \multicolumn{1}{c}{ Variable } & $\begin{array}{c}\text { Cultura Trabajo- } \\
\text { Familia }\end{array}$ & $\begin{array}{c}\text { Equilibrio Trabajo-Familia } \\
\text { Satisfacción } \\
\text { vital }\end{array}$ & $\begin{array}{c}\text { Felicidad } \\
\text { subjetiva }\end{array}$ \\
\hline Cultura Trabajo- Familia & - & & \\
Equilibrio Trabajo Familia & $.32^{* *}$ & - & - \\
Satisfacción vital & $-.21^{*}$ & -.09 & -.12 \\
Felicidad subjetiva & $-.23^{*}$ & $56^{* *}$ & - \\
\hline
\end{tabular}

$* p<.05$, dos colas. ${ }^{* *} p<.01$, dos colas.

Además, existe una correlación negativa baja estadísticamente significativa entre satisfacción vital y cultura trabajo familia (rho $=-.21$ ). De igual forma, las variables felicidad subjetiva y cultura trabajo-familia presentan una correlación negativa baja estadísticamente significativa (rho $=-.23, p=.019$ ); es decir, existe una correlación negativa baja entre cultura trabajo-familia y bienestar subjetivo, variable compuesta por satisfacción vital y felicidad subjetiva.

Como se observa en la tabla 4, la variable cultura trabajo familia es explicada en un $44.7 \%$ por las dimensiones de equilibrio trabajo familia, bienestar subjetivo y felicidad subjetiva. Asimismo, es importante saber qué porcentaje del modelo propuesto explica cada una de las variables y/o dimensiones anteriormente mencionadas. 
Tabla 4

Análisis de regresión sobre la variable cultura trabajo-familia

\begin{tabular}{ccccc}
\hline Modelo & $\mathrm{R}$ & $\mathrm{R}$ cuadrado & $\mathrm{R}$ cuadrado corregida & $\begin{array}{c}\text { Error típico de la } \\
\text { estimación }\end{array}$ \\
\hline 1 & $.669^{\mathrm{a}}$ & .447 & .419 & 11.976 \\
\hline
\end{tabular}

avariables predictoras: (Constante), Dimensiones de Trabajo-Familia y Bienestar.

Como se puede observar en la tabla 5, la interacción negativa trabajo-familia explica en un 44\% la cultura trabajo-familia; es decir, las variables felicidad subjetiva, satisfacción vital y las demás interfaz de equilibrio trabajo-familia explican un $0.7 \%$ del modelo de regresión propuesto.

Tabla 5

Análisis de regresión lineal del modelo Interfaz negativa trabajo-familia como predictor de cultura trabajo-familia

\begin{tabular}{ccccc}
\hline Modelo & $\mathrm{R}$ & R cuadrado & R cuadrado corregida & $\begin{array}{c}\text { Error típico de la } \\
\text { estimación }\end{array}$ \\
2 & $.663^{\mathrm{a}}$ & .440 & .435 & 11.812 \\
\hline
\end{tabular}

aariables predictoras: (constante), negativa trabajo-familia.

\section{Discusión}

El principal objetivo de la presente investigación es determinar la influencia del equilibrio trabajo-familia y el bienestar subjetivo en la cultura trabajo-familia de funcionarios del Poder Judicial de la Región del Maule. Se observa una relación significativa y positiva entre las variables cultura trabajo-familia y bienestar subjetivo, compuesto por satisfacción vital y felicidad subjetiva, lo que reafirma el supuesto de la incidencia de políticas organizacionales saludables para los empleados como lo representa el aumento de control de su vida, lo cual conlleva una reducción en los factores de estrés como contribución a la salud física y mental de los trabajadores. Es decir, los trabajadores de las instituciones que implementan políticas de trabajo y las ponen en práctica tienen una aumento en la satisfacción (Kossek et al., 2011).

Los resultados de la presente investigación corroboran la existencia de dicha relación de cultura trabajo familia y satisfacción vital. Esto podría deberse a la necesidad de considerar la integración entre el efecto de las prácticas a favor del equilibrio trabajo-familia y el bienestar subjetivo; además, la relación condicional de estas prácticas con una mejora en el bienestar en el trabajo no es incondicional o inevitable (Carvalho \& Chambel, 2016). De acuerdo con Clark (2000), al encontrarse en una cultura organizacional caracterizada por la formalidad y la jerarquía, se presentaría una relación negativa con el bienestar general que los empleados perciben a nivel organizacional. Por ende, existen otras variables que podrían afectar la correlación de las variables.

La cultura trabajo-familia cumple un rol importante dentro de las actitudes y las percepciones que generan los empleados acerca del apoyo que perciben de la organización para equilibrar la relación entre trabajo-familia; por lo tanto, una cultura de apoyo debería facilitar el logro del equilibrio trabajo familia 
de los empleados (Thompson et al., 1999). Los resultados respaldan lo anterior debido a que existe una relación significativa entre la cultura trabajo- familia y equilibrio trabajo-familia en los funcionarios del Poder Judicial de la región del Maule. Es importante mencionar que la correlación entre las dimensiones de equilibrio trabajo-familia y cultura trabajo-familia también es significativa con interfaz negativa trabajo-familia con interfaz negativa familia-trabajo e interfaz positiva trabajo-familia con interfaz positiva familia-trabajo.

Respecto a la hipótesis que plantea que existe una relación entre las variables bienestar subjetivo y equilibrio trabajo-familia, esta es esperable, porque tal como lo señala a Diener et al. (1999), las personas que presentan niveles adecuados de satisfacción en ambas esferas de su vida laboral y familiar experimentan mayores niveles de bienestar personal. Por lo tanto, quienes logran el equilibrio trabajo-familia mantienen mayores niveles de bienestar. En la misma línea, Jiménez y Moyano (2008) explican que los empleados perciben una mejor calidad de vida al lograr el equilibrio trabajo-familia y evitar los conflictos entre los diferentes roles de su vida.

Los resultados demuestran que existe una relación negativa, pero no significativa entre bienestar subjetivo (compuesto por satisfacción vital y felicidad subjetiva) y equilibrio trabajo-familia en funcionarios del Poder Judicial de la región del Maule. Esto podría deberse a que la relación entre equilibrio trabajo-familia y bienestar subjetivo es explicada por el bienestar en el trabajo, el cual se define como evitar el síndrome de burnout y aumentar la presencia de compromiso (Carvalho \& Chambel, 2016). Además, ambas variables afectan la percepción de los empleados acerca de los sistemas de trabajo de alto rendimiento percibido (Carvalho \& Chambel, 2016).

Se observa que al separar las dimensiones que componen el equilibrio trabajo-familia estas sí correlacionan significativamente con las dimensiones de bienestar subjetivo (felicidad subjetiva y satisfacción vital), interacción negativa trabajo-familia correlaciona significativamente con satisfacción vital, interacción negativa familia-trabajo, interacción positiva trabajo-familia e interacción positiva familia-trabajo. Las esferas negativas de la variable equilibrio trabajo-familia miden el conflicto trabajo familia y las esferas positivas miden conciliación (Greenhaus \& Beutell, 1985; Frone, 2003; Grzywacz \& Bass, 2003; Jiménez \& Moyano, 2008), lo cual explicaría la correlación negativa de las esferas negativas, porque a mayor conflicto menor bienestar subjetivo y la correlación de las esferas positivas es positiva, puesto que a mayor conciliación trabajo-familia, mayor bienestar subjetivo.

Además, y siguiendo lo anteriormente expuesto, Jiménez et al. (2009) explican que, si bien estas variables se correlacionan, los componentes de la interacción negativa con la interacción positiva son independientes entre sí al explicar los resultados obtenidos en la presente investigación.

Respecto a la hipótesis que define la existencia del modelo predictor de cultura trabajo familia, se confirma la influencia de las variables bienestar subjetivo (integradas por felicidad subjetiva y satisfacción vital) y equilibrio trabajo-familia compuesta por sus cuatro dimensiones: interacción negativa trabajo-familia, interacción negativa familia-trabajo, interacción positiva trabajo-familia e interacción positiva familia-trabajo.

Para que exista un buen equilibrio trabajo-familia, se deben promover tres pilares fundamentales: el individual, el Estado y la empresa. En el plano individual, el trabajador debe implementar sus propias 
estrategias que le permitan adaptar el ámbito familiar y el laboral, mientras que, por parte del Estado, se debe velar por los intereses familiares al diseñar un marco legal mediante políticas públicas que favorezcan el equilibrio entre familia-trabajo.

Por último, se encuentra el ámbito empresarial, el cual debe asegurar el bienestar de los empleados en conjunto con un ambiente grato que les permita a los trabajadores equilibrar estas dos esferas, tanto el área laboral como el área familiar (Debeljuh \& Jáuregui, 2004). Ello demuestra que existe una relación entre el equilibrio trabajo-familia, la cultura trabajo-familia al interior de la organización e impuesta por el Estado y el bienestar del trabajador.

Los resultados demostraron que las variables bienestar subjetivo y equilibrio trabajo-familia explican en un $44.7 \%$ la cultura trabajo-familia. Los resultados generales obtenidos en la presente investigación contribuyen a confirmar las predicciones del modelo de cultura trabajo-familia utilizado (Thompson et al., 1999) y la incorporación de la dimensión del interfaz negativa familia-trabajo como principal predictor en este modelo de la cultura trabajo-familia.

En conclusión, para medir la cultura trabajo-familia es indispensable incorporar la interacción negativa trabajo-familia. Al existir una correlación significativa entre las variables, se puede hipotetizar que la interacción negativa trabajo-familia incorpora a la demás variables, puesto que están relacionadas y produce una disminución en el porcentaje de explicación de las demás variables en el modelo.

Los funcionarios de la institución, a pesar de tener conflictos trabajo-familia, perciben apoyo de la organización para lograr el equilibrio. Esto podría deberse a características de la institución, características personales de cada funcionario, el temor al ser identificados al expresar disconformidad, entre otras. Por lo tanto, se recomienda, para futuras investigaciones, profundizar las razones de la relación causal de interacción negativa trabajo-familia y cultura trabajo-familia.

Los resultados generales obtenidos en la presente investigación contribuyen a confirmar las predicciones del modelo de cultura trabajo-familia utilizado (Thompson et al., 1999) y la incorporación de la dimensión del interfaz negativa familia- trabajo como principal predictor en este modelo de la cultura trabajo-familia.

En cuanto a las otras variables, se recomienda seguir investigando en cuanto a los valores predictores del bienestar subjetivo y el equilibrio trabajo-familia tienen sobre la cultura trabajo-familia, pues si bien la interfaz negativa trabajo-familia predice, en mayor grado, las otras variables se correlacionan y explican el modelo en un porcentaje bajo; por lo tanto, en futuras investigaciones se deben considerar los datos obtenidos para obtener un análisis más exhaustivo de los datos.

Finalmente, en futuros estudios, se recomienda incorporar variables como la comunicación entre los directivos y los funcionarios, debido a que pueden existir políticas y apoyo de los directivos, pero si estas no se comunican de la manera adecuada, los empleados no las perciben (Kirby \& Krone, 2002), al igual que el clima laboral, debido a que si existe un mal clima organizacional este dificultará la implementación y el uso de las políticas familiarmente responsables, lo que desfavorece a la cultura trabajo-familia al interior de la organización (McDonald et al., 2005). En ese sentido, si existiese un mal clima este repercutirá de manera directa en el área laboral, lo que explicaría en cierta parte el bajo uso de las 
CULTURA TRABAJO-FAMILIA, EQUILIBRIO TRABAJO-FAMILIA, BIENESTAR EN TRABAJADORES

políticas existentes. Esta limitación puede enfrentarse en la medida que haya una detección de políticas, procedimientos y protocolos que puedan incidir en los indicadores estudiados.

\section{Referencias}

Álvarez, M. (2015). Políticas públicas del cuidado con corresponsabilidad. Revista Novedades en Población, 11(21) 130-136.

Behson, S. (2002). Which Dominates? The Relative Importance of Work-Family Organizational Support and General Organizational Context on Employee Outcomes. Journal of Vocational Behavior, 61(1), 53-72.

Brizuela, P. (2016). Calidad de vida y nivel de actividad fisica en trabajadores que asisten al servicio de salud del poder judicial con factores de riesgo asociados a síndrome metabólico [Tesis de maestría, Universidad Nacional, Costa Rica]. Universidad Nacional, Costa Rica, Archivo Digital. https://repositorio.una.ac.cr/ handle/11056/14048

Caamaño, E. (2008). El permiso parental y la progresiva inclusión del padre en los derechos para la armonización del trabajo y la vida familiar. Revista de Derecho de la Pontificia Universidad Católica de Valparaíso, 31, 325-354.

Cachutt, C., \& Ortiz, F. (2015). Medición de la integración trabajo-familia en organizaciones. Revista de la Facultad de Ingeniería Industrial, 18(2), 7-13. https://www.researchgate.net/ publication/307180377_Medicion_de_la_integracion_trabajo-familia_en_organizaciones

Carvalho, V., \& Chambel, M, (2016). Perceived High-Performance Work Systems and Subjective Well-Being: Work-to-Family Balance and Well-Being at Work as Mediators. Journal of Career Development, 43(2), 116-129.

Clark, S. (2000). Work/family border theory: A new theory of work/family balance. Human relations, 53(6), 747-770.

Debeljuh, P., \& Jáuregui, K. (2004). Trabajo y familia: hacia una cultura familiar amigable en el contexto latinoamericano. Journal of Economics, Finance and Administrative Science, 9(16), 91-102.

Diener, E., Suh, E.M., Lucas, R. \& Smith, H. (1999). Subjective Well-Being: Three Decades of Progress. Psychological Bulletin, 125(2), 276-302.

Faúndez, A., Peyrin, C., \& Weinstein, M. (2013). Guía sistema de gestión de igualdad de género y conciliación de la vida laboral, familiar y personal en las organizaciones. Recuperado el 23 de octubre de 2013, de https://www.minmujeryeg.cl/ministerio/estructura/mujer-y-trabajo/ buenas-practicas-laborales-y-trabajo-decente-para-la-igualdad-de-genero/

Frone, M. (2003). Work-family balance. En J.C. Quick \& L.E. Tetrick, Handbook of occupational health psychology, (pp. 143-162). American Psychological Association

Gómez, V., \& Jiménez, A. (2019). Género y trabajo: hacia una agenda nacional de equilibrio trabajo-familia en Chile. Convergencia Revista de Ciencias Sociales, 26(79), 1-24. 


\section{A. JIMÉNEZ Y V. GÓMEZ}

Gragnano, A., Simbula, S., \& Miglioretti, M. (2020). Work-Life balance: Weighing the importance of workfamily and work-health balance. Int. J. Environ. Res. Public Health 17(907),1-20. https://doi.org/10.3390/ ijerph17030907

Guic, E., Mora, P., Rey, R., \& Robles, A. (2006). Estrés organizacional y salud en funcionarios de centros de atención primaria de una comuna de Santiago. Revista médica de Chile, 134(4), 447-455.

Grant-Vallone, E., \& Donaldson, S. (2001). Consequences of work-family conflict on employee well-being over time. Work \& stress, 15(3), 214-226.

Greenhaus, J., \& Beutell, N. (1985). Sources of conflict between work and family roles. Academy of Management Review, 10(1), 76-88.

Greenhaus, J., Collins, K., \& Shaw, J. (2003). The relation between work-family balance and quality of life. Journal of vocational behavior, 63(3), 510-531.

Grzywacz, J. G., \& Bass, B. L. (2003). Work, family, and mental health: Testing different models of work-family fit. Journal of Marriage and Family, 65(1), 248-261.

Gröpel, P., \& Kuhl, J. (2009). Work-life balance and subjective well-being: The mediating role of need fulfilment. British Journal of Psychology, 100(2), 365-375.

Instituto Nacional de Estadística de Chile. (2017). Encuesta Nacional sobre Uso del Tiempo. Principales Resultados. Recuperado el 22 de septiembre de 2017, de http://historico.ine.cl/enut/files/principales_resultados/ documento_resultados_ENUT.pdf

Jiménez, A., Concha, M., \& Zúñiga, R. (2012). Conflicto trabajo-familia, autoeficacia parental y estilos parentales percibidos en padres y madres de la ciudad de Talca, Chile. Acta Colombiana de Psicología, 15(1), 57-65.

Jiménez, A., \& Gómez, V. (2015). Conciliando trabajo-familia: análisis desde la perspectiva de género. Diversitas, 11(2), 289-302. https://revistas.usantotomas.edu.co/index.php/diversitas/article/ view/2681

Jiménez, A., \& Moyano, E. (2008). Factores laborales de equilibrio entre trabajo y familia: medios para mejorar la calidad de vida. Universum, 23(1), 116-133.

Jiménez, B., Vergel, A., Muñoz, A., \& Geurts, S. (2009). Propiedades psicométricas de la versión española del Cuestionario de Interacción Trabajo-Familia (SWING). Psicothema, 21(2), 331-337.

Kirby, E., \& Krone, K. (2002). “The policy exists but you can’t really use it”: communication and the structuration of work-family policies. Journal of Applied Communication Research, 30(1), 50-77.

Kossek, E., Baltes, B., \& Matthews, R. (2011). Toward a review and reconceptualization of the work/family literature. Industrial \& Organizational Psychology, 4(3), 352-369.

Lyubomirsky, S., \& Lepper, H. (1999). A measure of subjective happiness: Preliminary reliability and construct validation. Social Indicators Research, 46, 137-155.

McDonald, P., Brown, K., \& Bradley, L. (2005). Explanations for the provision-utilisation gap in work-life policy. Women in Management Review, 20(1), 37-55. 
CULTURA TRABAJO-FAMILIA, EQUILIBRIO TRABAJO-FAMILIA, BIENESTAR EN TRABAJADORES

Ministerio de Salud de Chile. (2017). Protocolo de vigilancia de riesgos psicosociales en el Trabajo. Recuperado el 23 de marzo de 2017, de https://dipol.minsal.cl/departamentos-2/salud-ocupacional/riesgos-psicosociales/

Moreno, B., Sanz, A., Rodríguez, A., \& Geurts, S. (2009) Propiedades psicométricas de la versión española del Cuestionario de Interacción Trabajo-Familia (SWING). Psicothema, 21, 331-337.

Moyano, E., \& Ramos, N. (2007). Bienestar subjetivo: midiendo satisfacción vital, felicidad y salud en población chilena de la Región Maule. Universum (Talca), 22(2), 177-193.

Riquelme, E., Rojas, A., \& Jiménez, A. (2012). Equilibrio trabajo-familia, apoyo familiar, autoeficacia parental y funcionamiento familiar percibidos por funcionarios públicos de Chile. Trabajo y sociedad: Indagaciones sobre el empleo, la cultura y las prácticas políticas en sociedades segmentadas, (18)13, 203-215.

Schmidt, Vivien (2014). La política de los pactos sociales y la protección social: ¿importa el discurso? Políticas sociales, (178). Recuperado el 23 de junio de 2014, de http://repositorio.cepal.org/bitstream/ handle/11362/35902/1/S2013402_es.pdf

Staab, S. (2017). Gender and the Politics of Gradual Change: Social Policy Reform and Innovation in Chile. Palgrave Macmillan.

Servicio Nacional de la Mujer (2013). Guía: Sistemas de Gestión de Igualdad de Género y Conciliación de la Vida Laboral, Familiar y Personal en las Organizaciones, Noviembre 2013. https://energia.gob.cl/sites/default/ files/documentos/guia_implementacion_norma_nch3262.pdf

Servicio Nacional de la Mujer (2003). Análisis de los costos y beneficios de implementar medidas de conciliación vida laboral y familiar en la empresa. http://estudios.sernam.cl/documentos/?eODYxNjMwAn\%C3\%A1lisis de los_costos_y_beneficios_de implementar_medidas_de_concliliaci $\% \mathrm{C} 3 \% \mathrm{~B} 3 \mathrm{n} \_$de la la vida_laboral_y familiar_de_las_empresas

Smith, J., \& Gardner, D. (2007). Factors Affecting Employee Use of Work-Life Balance Initiatives. New Zealand Journal of Psychology, 36(1), 3-12.

Spielberg C., \& Reheiser E. (1998). Encuesta de estrés laboral: Diferencias de género en la medición del estrés ocupacional. Revista de Psicología, III(3). https://sisbib.unmsm.edu.pe/BVrevistas/psicologia/1998_n3 I encuesta_estres.htm

Thompson, C., Beauvais, L., \& Lyness, K. (1999). When work-family benefits are not enough: The influence of work-family culture on benefit utilization, organizational attachment, and work-family conflict. Journal of vocational behavior, 54(3), 392-415.

Recibido: 3 de agosto de 2018 Revisión recibida: 15 de marzo de 2021 Aceptado: 22 de abril de 2021 
Sobre el autor y la autora:

Andrés Eduardo Jiménez Figueroa labora como profesor investigador en la Facultad de Psicología de la Universidad de Talca (Chile). Se tituló como psicólogo organizacional (Universidad de Santiago, Chile), magíster en Dirección y Administración de Recursos Humanos (Universidad de Santiago, Chile) y doctor en Administración de Empresas (Universidad Central de Nicaragua). Responsable de la línea de investigación calidad de vida laboral y conciliación trabajo-familia. Participa en grupo de investigación en calidad de vida y ambientes saludables y el grupo de investigación (Universidad de Talca, Chile) y "Nuevas subjetividades, sociabilidades y configuraciones familiares". Cuenta con más de 40 publicaciones, comunicaciones y conferencias en congresos nacionales e internacionales.

Verónica Gómez Urrutia es doctora en Sociología. Trabaja como profesora de la Universidad Autónoma de Chile e investigadora del Instituto Iberoamericano de Desarrollo Sostenible. Se desempeña como directora del Grupo de Investigación "Nuevas subjetividades, sociabilidades y configuraciones familiares" en la misma casa de estudios. Ha sido responsable de proyectos financiados por la Agencia Nacional de Investigación y Desarrollo de Chile (ANID). Cuenta con numerosas publicaciones en el área de conciliación trabajo-familia.

Publicado en línea: 30 de junio de 2021

Revista Costarricense de Psicología

ISSN 0257-1439 / ISSNe 1659-2913

2021, Vol. 40, N. ${ }^{\circ} 1$ 